\title{
INEQUALITIES FOR THE WASSERSTEIN MEAN OF POSITIVE DEFINITE MATRICES
}

\author{
RAJENDRA BHATIA, TANVI JAIN, AND YONGDO LIM
}

\author{
Dedicated to Fumio Hiai, and to the memory of Denes Petz
}

\begin{abstract}
We prove majorization inequalities for different means of positive definite matrices. These include the Cartan mean (the Karcher mean), the log Euclidean mean, the Wasserstein mean and the power mean.
\end{abstract}

\section{INTRODUCTION}

Let $\mathbb{P}$ be the space of $n \times n$ complex positive definite matrices. The Bures-Wasserstein distance on $\mathbb{P}$ is the metric defined as

$$
d(A, B)=\left[\operatorname{tr}(A+B)-2 \operatorname{tr}\left(A^{1 / 2} B A^{1 / 2}\right)^{1 / 2}\right]^{1 / 2} .
$$

Let $A_{1}, \ldots, A_{m}$ be given positive definite matrices and let $w=\left(w_{1}, \ldots, w_{m}\right)$ be a vector of weights; i.e., $w_{j} \geq 0$ and $\sum_{j=1}^{m} w_{j}=1$. Then the (weighted) Wasserstein mean, or the Wasserstein barycentre of $A_{1}, \ldots, A_{m}$ is defined as

$$
\Omega\left(w ; A_{1}, \ldots, A_{m}\right)=\underset{X \in \mathbb{P}}{\operatorname{argmin}} \sum_{j=1}^{m} w_{j} d^{2}\left(X, A_{j}\right) .
$$

It can be shown that the function on $\mathbb{P}$ defined by the sum on the right hand side of (2) has a unique minimizer, and the notation argmin is used for this minimizer. It is also known that $\Omega$ is the unique positive definite solution of the equation

$$
X=\sum_{j=1}^{m} w_{j}\left(X^{1 / 2} A_{j} X^{1 / 2}\right)^{1 / 2} .
$$

In the special case $m=2$, writing $\left(A_{1}, A_{2}\right)=(A, B)$ and $\left(w_{1}, w_{2}\right)=$ $(1-t, t)$, where $0 \leq t \leq 1$, we have an explicit formula for $\Omega$. Denoting this by $A \diamond_{t} B$ we have

$$
A \diamond_{t} B=(1-t)^{2} A+t^{2} B+t(1-t)\left[(A B)^{1 / 2}+(B A)^{1 / 2}\right] .
$$

The matrix $A B$ has only positive eigenvalues and, therefore, has a unique square root with positive eigenvalues. This is the matrix $(A B)^{1 / 2}$ in the expression above. The metric $d$ in (1) is the distance corresponding to an underlying Riemannian metric on $\mathbb{P}$, and (4) is an equation for the geodesic

2010 Mathematics Subject Classification. 15A42, 15A18, 47A64, 47A30.

Key words and phrases. Positive definite matrices, Cartan mean, Wasserstein mean, log Euclidean mean, majorisation, unitarily invariant norm. 
segment joining two points $A$ and $B$ in the manifold $\mathbb{P}$. The special choice $t=1 / 2$ gives the midpoint of this geodesic. This is denoted by

$$
A \diamond B=\frac{1}{4}\left[A+B+(A B)^{1 / 2}+(B A)^{1 / 2}\right]
$$

and can be thought of as the Wasserstein mean of $A$ and $B$.

We refer the reader to our recent article [11] for the relevance and importance of the Wasserstein metric, mean and barycentre in various areas like quantum information, statistics, optimal transport and Riemannian geometry.

More familiar in the matrix theory literature, and much studied in the past few years, has been the geometric mean, variously called the Cartan mean, the Karcher mean or the Riemannian mean. To describe this we start with the Cartan metric

$$
\delta(A, B)=\left\|\log A^{-1 / 2} B A^{-1 / 2}\right\|_{2},
$$

where $\|X\|_{2}=\left(\operatorname{tr} X^{*} X\right)^{1 / 2}$ is the Frobenius norm on matrices. The weighted Cartan mean (or the weighted geometric mean) of $A_{1}, \ldots, A_{m}$ is defined as

$$
G\left(w ; A_{1}, \ldots, A_{m}\right)=\underset{X \in \mathbb{P}}{\operatorname{argmin}} \sum_{j=1}^{m} w_{j} \delta^{2}\left(X, A_{j}\right) .
$$

The (unique) solution of this minimization problem is also the positive definite solution of the equation

$$
\sum_{j=1}^{m} w_{j} \log \left(X^{-1 / 2} A_{j} X^{-1 / 2}\right)=0 .
$$

The mean $G$ was introduced in matrix analysis by M. Moakher [21] and R. Bhatia and J. Holbrook [9] as the solution of the long standing problem of defining an appropriate geometric mean of several positive definite matrices, and has since then been an object of intense study.

Analogous to (4) the equation of the geodesic segment joining $A$ and $B$ with respect to the metric $\delta$ is

$$
A \#_{t} B=A^{1 / 2}\left(A^{-1 / 2} B A^{-1 / 2}\right)^{t} A^{1 / 2}, \quad 0 \leq t \leq 1 .
$$

This is also called the $t$-weighted geometric mean of $A$ and $B$. When $t=1 / 2$, this reduces to

$$
A \# B=A^{1 / 2}\left(A^{-1 / 2} B A^{-1 / 2}\right)^{1 / 2} A^{1 / 2},
$$

and is called the geometric mean of $A$ and $B$.

Presented with the two important means $\Omega$ and $G$, arising from two different geometries on $\mathbb{P}$, it is natural to ask for comparisons between them. The two metrics $d$ and $\delta$ are strikingly different. Endowed with the metric $d$, the manifold $\mathbb{P}$ has nonnegative curvature [22, 11]; with the metric $\delta$ it has nonpositive curvature [7]. The Cartan mean has nice order properties: it is monotonic in variables $A_{1}, \ldots, A_{m}$ [18] and lies between the harmonic and 
arithmetic means; i.e.,

$$
\left(\sum_{j=1}^{m} w_{j} A_{j}^{-1}\right)^{-1} \leq G\left(w ; A_{1}, \ldots, A_{m}\right) \leq \sum_{j=1}^{m} w_{j} A_{j}
$$

where $\leq$ is the Löwner ordering; $A \leq B$ if $B-A$ is positive semidefinite. It has been shown in [11] that the Wasserstein mean $\Omega$ is not monotonic in the variables $A_{1}, \ldots, A_{m}$. While it is always bounded above by the arithmetic mean, it may not be bounded below by the harmonic mean. A fortiori the operator inequality $G \leq \Omega$ does not hold. Somewhat surprisingly, good comparison theorems in terms of log majorizations can be proved, and that is the main purpose of this paper.

Let $x=\left(x_{1}, \ldots, x_{n}\right)$ and $y=\left(y_{1}, \ldots, y_{n}\right)$ be two $n$-tuples of nonnegative numbers. Let $x_{1}^{\downarrow} \geq x_{2}^{\downarrow} \geq \ldots \geq x_{n}^{\downarrow}$ be the decreasing rearrangement of $x_{1}, \ldots, x_{n}$. If for all $1 \leq k \leq n$

$$
\prod_{j=1}^{k} x_{j}^{\downarrow} \leq \prod_{j=1}^{k} y_{j}^{\downarrow}
$$

we say that $x$ is weakly log majorised by $y$, and write this as

$$
x \underset{w \log }{\prec} y .
$$

If, in addition to (12) we also have

$$
\prod_{j=1}^{n} x_{j}^{\downarrow}=\prod_{j=1}^{n} y_{j}^{\downarrow}
$$

we say $x$ is $\log$ majorised by $y$, and write this as

$$
x \underset{\log }{\prec} y \text {. }
$$

Let $\lambda(A)$ stand for the eigenvalue $n$-tuple of $A$. We will show that $\lambda(G) \underset{\log }{\prec} \lambda(\Omega)$. In fact, we will prove a stronger result that involves another mean called the log Euclidean mean. This is the matrix defined as

$$
L\left(w ; A_{1}, \ldots, A_{m}\right)=\exp \left(\sum_{j=1}^{m} w_{j} \log A_{j}\right) .
$$

If we replace the metric $\delta$ in (6) by

$$
\delta_{L}(A, B)=\|\log A-\log B\|_{2},
$$

and consider the corresponding minimization problem (17), then the solution is the $\log$ Euclidean mean $L$. In the special case when $A_{1}, \ldots, A_{m}$ are pairwise commuting positive definite matrices, we have

$$
G=L=\prod_{j=1}^{m} A_{j}^{w_{j}} .
$$

The first half of the following theorem was proved by Hiai and Petz [17], the second is new. 
Theorem 1. Let $G=G\left(w ; A_{1}, \ldots, A_{m}\right), L=L\left(w ; A_{1}, \ldots, A_{m}\right)$ and $\Omega=$ $\Omega\left(w ; A_{1}, \ldots, A_{m}\right)$ be the Cartan, the log Euclidean, and the Wasserstein means of positive definite matrices $A_{1}, \ldots, A_{m}$. Then

$$
\lambda(G) \underset{\log }{\prec} \lambda(L) \underset{w \log }{\prec} \lambda(\Omega) .
$$

There is another family of means that is important in this context. The $p$-th power mean of $A_{1}, \ldots, A_{m}$ is defined as

$$
Q_{p}\left(w ; A_{1}, \ldots, A_{m}\right)=\left(\sum_{j=1}^{m} w_{j} A_{j}^{p}\right)^{1 / p}, \quad-\infty<p<\infty .
$$

For $p=0$, this quantity is to be interpreted as a limit. It was shown by Bhagwat and Subramanian [5] that

$$
\lim _{p \rightarrow 0^{ \pm}} Q_{p}\left(w ; A_{1}, \ldots, A_{m}\right)=L\left(w ; A_{1}, \ldots, A_{m}\right) .
$$

Of special interest to us here is the case $p=1 / 2$. When $A_{1}, \ldots, A_{m}$ commute we have

$$
\Omega\left(w ; A_{1}, \ldots, A_{m}\right)=\left(\sum_{j=1}^{m} w_{j} A_{j}^{1 / 2}\right)^{2}=Q_{1 / 2}\left(w ; A_{1}, \ldots, A_{m}\right) .
$$

Thus $\Omega$ and $Q_{1 / 2}$ can be regarded as two different noncommutative extensions of the same object, and for this reason it is natural to ask for comparisons between them.

We remark here that just as we have realized the means $\Omega, G$ and $L$ as solutions of least square problems for certain metrics on $\mathbb{P}$, so can be done for $Q_{1 / 2}$. The Hellinger distance or the Bhattacharya distance between probability vectors $p=\left(p_{1}, \ldots, p_{n}\right)$ and $q=\left(q_{1}, \ldots, q_{n}\right)$ is defined as

$$
d(p, q)=\left\|p^{1 / 2}-q^{1 / 2}\right\|_{2}=\left[\sum_{i=1}^{n}\left(\sqrt{p_{i}}-\sqrt{q_{i}}\right)^{2}\right]^{1 / 2} .
$$

A straightforward extension to positive definite matrices is the distance

$$
d_{H}(A, B)=\left\|A^{1 / 2}-B^{1 / 2}\right\|_{2}=\left[\operatorname{tr}(A+B)-2 \operatorname{tr} A^{1 / 2} B^{1 / 2}\right]^{1 / 2} .
$$

Compare this with the Bures-Wasserstein distance (11). It can be seen that the solution to the least squares problem (2) when $d$ is replaced by $d_{H}$ is the mean $Q_{1 / 2}\left(w ; A_{1}, \ldots, A_{m}\right)$.

For the comparison between the means $Q_{1 / 2}$ and $\Omega$ we have the following result.

Theorem 2. For all positive definite matrices $A_{1}, \ldots, A_{m}$ and weights $w_{1}, \ldots, w_{m}$, we have

$$
\left\|Q_{1 / 2}\left(w ; A_{1}, \ldots, A_{m}\right)\right\|_{p} \leq\left\|\Omega\left(w ; A_{1}, \ldots, A_{m}\right)\right\|_{p},
$$

for the Schatten $p$-norms with $p=1$ and $\infty$. In the case $m=2$, the inequality (24) holds also with $p=2$. 
Theorem 2 establishes a part of the following:

Conjecture 1 The inequality (24) is valid, more generally, for all unitarily invariant norms. In other words, we have the weak majorisation

$$
\lambda\left(Q_{1 / 2}\right) \underset{w}{\prec} \lambda(\Omega) .
$$

We remark that when $m=2$, and $\left(w_{1}, w_{2}\right)=(1 / 2,1 / 2)$ the conjecture says that

$$
\left\|\left|A+B+A^{1 / 2} B^{1 / 2}+B^{1 / 2} A^{1 / 2}\left\|\left|\leq\left\|\mid A+B+(A B)^{1 / 2}+(B A)^{1 / 2}\right\| \|,\right.\right.\right.\right.
$$

for every unitarily invariant norm, and Theorem 2 includes the statement that this is true for the $p$-norms with $p=1,2, \infty$. There is a parallel here with results proved in [12] which we discuss later in Section 3.

\section{ProOfS}

The first majorisation in (19) is mentioned as a remark at the end of the paper [17]. Some of the ideas needed for the proofs of the two majorisations are same. Therefore, for the reader's convenience we include here proofs of both the majorisations in (19) and also of the propositions that go into them (with some simplifications). The main ideas originate in the work of the Japanese school beginning with T. Ando [2], followed by T. Ando and Hiai [3], and then by J. I. Fujii, M. Fujii, Y. Seo [14] and T. Yamazaki [23].

The function $f(A)=A^{p}$ on positive definite matrices is operator convex if $1 \leq p \leq 2$, and operator concave if $0 \leq p \leq 1$. Operator convexity and concavity are characterized by Jensen type inequalities called Hansen's inequalities [15] and [16, Theorem 2.1], which say that for every contraction $X$ we have

$$
\left(X^{*} A X\right)^{p} \leq X^{*} A^{p} X, \quad \text { if } 1 \leq p \leq 2,
$$

and

$$
\left(X^{*} A X\right)^{p} \geq X^{*} A^{p} X, \quad \text { if } 0 \leq p \leq 1 .
$$

A standard technique in proving log majorisations like (19) is the use of antisymmetric tensor powers. This is so because if $\Lambda^{k} A$ denotes the $k$ th antisymmetric tensor power of $A$, then

$$
\prod_{j=1}^{k} \lambda_{j}^{\downarrow}(A)=\lambda_{1}^{\downarrow}\left(\Lambda^{k} A\right), \quad 1 \leq k \leq n .
$$

The map $A \mapsto \Lambda^{k} A$ is multiplicative, i.e., $\Lambda^{k}(A B)=\left(\Lambda^{k} A\right)\left(\Lambda^{k} B\right)$ and $\Lambda^{k} A^{p}=\left(\Lambda^{k} A\right)^{p}, p \in(-\infty, \infty)$. So the geometric mean $G$ is well disposed towards tensor powers. It is clear from (9) that $\Lambda^{k}\left(A \#_{t} B\right)=\left(\Lambda^{k} A\right) \#_{t}\left(\Lambda^{k} B\right)$. The same property holds for the several variable geometric mean

$$
\Lambda^{k} G\left(w ; A_{1}, \ldots, A_{m}\right)=G\left(w ; \Lambda^{k} A_{1}, \ldots, \Lambda^{k} A_{m}\right) .
$$

See [10] for a proof. Most of the other means do not behave as well with respect to tensor powers. For the log Euclidean mean this difficulty is circumvented 
through the following ingenious proposition, proved by Ando and Hiai [3] for two variables and extended by Fujii et al [14] to several variables.

Proposition 3. We have

$$
\lim _{p \rightarrow 0^{+}} G\left(w ; A_{1}^{p}, \ldots, A_{m}^{p}\right)^{1 / p}=L\left(w ; A_{1}, \ldots, A_{m}\right) .
$$

Proof. By the arithmetic-geometric-harmonic mean inequalities (11), we have for every $p>0$

$$
\left(\sum_{j=1}^{m} w_{j} A_{j}^{-p}\right)^{-1} \leq G\left(w ; A_{1}^{p}, \ldots, A_{m}^{p}\right) \leq \sum_{j=1}^{m} w_{j} A_{j}^{p}
$$

Take logarithms and use the fact that $\log$ is an operator monotone function to get

$$
-\log \left(\sum_{j=1}^{m} w_{j} A_{j}^{-p}\right) \leq \log G\left(w ; A_{1}^{p}, \ldots, A_{m}^{p}\right) \leq \log \left(\sum_{j=1}^{m} w_{j} A_{j}^{p}\right) .
$$

Multiplying by $1 / p$ we get

$$
\log \left(\sum_{j=1}^{m} w_{j} A_{j}^{-p}\right)^{-1 / p} \leq \log G\left(w ; A_{1}^{p}, \ldots, A_{m}^{p}\right)^{1 / p} \leq \log \left(\sum_{j=1}^{m} w_{j} A_{j}^{p}\right)^{1 / p} .
$$

Now take the limit as $p \rightarrow 0^{+}$and use the result of Bhagwat and Subramanian (21) to obtain (30).

Using the equation (8) that characterizes $G$, we see that $G\left(w ; A_{1}, \ldots, A_{m}\right)=$ $I \Longleftrightarrow \sum_{j=1}^{m} w_{j} \log A_{j}=0 \Longleftrightarrow \sum_{j=1}^{m} w_{j} \log A_{j}^{p}=0$ for all $p>0 \Longleftrightarrow$ $G\left(w ; A_{1}^{p}, \ldots, A_{m}^{p}\right)=I$ for all $p>0$. The following proposition handles the case $G \leq I$. It was proved for two variables by Ando [2] and for general $m$ by Yamazaki [23].

Proposition 4. Suppose $G\left(w ; A_{1}, \ldots, A_{m}\right) \leq I$. Then

$$
G\left(w ; A_{1}^{p}, \ldots, A_{m}^{p}\right) \leq G\left(w ; A_{1}, \ldots, A_{m}\right), \quad 1 \leq p<\infty,
$$

and

$$
G\left(w ; A_{1}^{p}, \ldots, A_{m}^{p}\right) \geq G\left(w ; A_{1}, \ldots, A_{m}\right), \quad 0 \leq p \leq 1 .
$$

Proof. Let $A_{1}, \ldots, A_{m} \in \mathbb{P}$, and let $X=G\left(w ; A_{1}, \ldots, A_{m}\right)$. By the congruence invariance property of $G$

$$
G\left(w ; X^{-1 / 2} A_{1} X^{-1 / 2}, \ldots, X^{-1 / 2} A_{m} X^{-1 / 2}\right)=I .
$$

So, by the remark preceding Proposition 4

$$
G\left(w ;\left(X^{-1 / 2} A_{1} X^{-1 / 2}\right)^{p}, \ldots,\left(X^{-1 / 2} A_{m} X^{-1 / 2}\right)^{p}\right)=I
$$

for all $p>0$.

If $X \geq I$, then $X^{-1 / 2} \leq I$. Then by Hansen's inequality (27), for $1 \leq p \leq$ 2 we have $\left(X^{-1 / 2} A_{j} X^{-1 / 2}\right)^{p} \leq X^{-1 / 2} A_{j}^{p} X^{-1 / 2}$. The monotonicity property of $G$ then gives

$$
I \leq G\left(w ; X^{-1 / 2} A_{1}^{p} X^{-1 / 2}, \ldots, X^{-1 / 2} A_{m}^{p} X^{-1 / 2}\right),
$$


and the congruence invariance of $G$ shows that

$$
X \leq G\left(w ; A_{1}^{p}, \ldots, A_{m}^{p}\right) .
$$

We have shown that if $G\left(w ; A_{1}, \ldots, A_{m}\right) \geq I$, then $G\left(w ; A_{1}, \ldots, A_{m}\right) \leq$ $G\left(w ; A_{1}^{p}, \ldots, A_{m}^{p}\right)$ for $1 \leq p \leq 2$.

If $G\left(w ; A_{1}, \ldots, A_{m}\right) \leq I$, then $G\left(w ; A_{1}^{-1}, \ldots, A_{m}^{-1}\right) \geq I$. So, by what we have proved $G\left(w ; A_{1}^{-1}, \ldots, A_{m}^{-1}\right) \leq G\left(w ; A_{1}^{-p}, \ldots, A_{m}^{-p}\right)$ for $1 \leq p \leq 2$. Inverting again we get the inequality (31) for $1 \leq p \leq 2$. Using this the validity of the inequality can be established for $2 \leq p \leq 4$, and successively for intervals beyond. The same arguments can be used to prove (32).

We now turn to the proof of Theorem 1. All the means under consideration here (arithmetic, geometric, harmonic, $\log$ Euclidean, Wasserstein, and the $p$ th power mean) are homogeneous in variables $A_{j}$; i.e., for all $\alpha>0$ we have

$$
G\left(w ; \alpha A_{1}, \ldots, \alpha A_{m}\right)=\alpha G\left(w ; A_{1}, \ldots, A_{m}\right),
$$

etc. So, to prove an inequality like $\lambda_{1}^{\downarrow}(G) \leq \lambda_{1}^{\downarrow}(L)$ it suffices to show that $G \leq I$ whenever $L \leq I$.

Let $0<p<1$ and suppose

$$
G\left(w ; A_{1}^{p}, \ldots, A_{m}^{p}\right)^{1 / p} \leq I .
$$

Then

$$
G\left(w ; A_{1}^{p}, \ldots, A_{m}^{p}\right) \leq I .
$$

It follows from Proposition 4 that

$$
G\left(w ; A_{1}, \ldots, A_{m}\right)=G\left(w ;\left(A_{1}^{p}\right)^{1 / p}, \ldots,\left(A_{m}^{p}\right)^{1 / p}\right) \leq G\left(w ; A_{1}^{p}, \ldots, A_{m}^{p}\right) \leq I .
$$

Hence, for all $0<p<1$ we have

$$
\lambda_{1}^{\downarrow}\left(G\left(w ; A_{1}, \ldots, A_{m}\right)\right) \leq \lambda_{1}^{\downarrow}\left(G\left(w ; A_{1}^{p}, \ldots, A_{m}^{p}\right)^{1 / p}\right) .
$$

Replacing $A_{j}$ by $\Lambda^{k}\left(A_{j}\right)$ and using (29) we get

$$
\begin{aligned}
\prod_{j=1}^{k} \lambda_{j}^{\downarrow}\left(G\left(w ; A_{1}, \ldots, A_{m}\right)\right) & =\lambda_{1}^{\downarrow}\left(\Lambda^{k} G\left(w ; A_{1}, \ldots, A_{m}\right)\right) \\
& =\lambda_{1}^{\downarrow}\left(G\left(w ; \Lambda^{k} A_{1}, \ldots, \Lambda^{k} A_{m}\right)\right) \\
& \leq \lambda_{1}^{\downarrow}\left(G\left(w ;\left(\Lambda^{k} A_{1}\right)^{p}, \ldots,\left(\Lambda^{k} A_{m}\right)^{p}\right)^{1 / p}\right) \\
& =\lambda_{1}^{\downarrow}\left(G\left(w ;\left(\Lambda^{k} A_{1}^{p}\right), \ldots,\left(\Lambda^{k} A_{m}^{p}\right)\right)^{1 / p}\right) \\
& =\lambda_{1}^{\downarrow}\left(\Lambda^{k} G\left(w ; A_{1}^{p}, \ldots, A_{m}^{p}\right)^{1 / p}\right) \\
& =\prod_{j=1}^{k} \lambda_{j}^{\downarrow}\left(G\left(w ; A_{1}^{p}, \ldots, A_{m}^{p}\right)^{1 / p}\right)
\end{aligned}
$$

for all $1 \leq k \leq n$. Letting $p \rightarrow 0$ we get from Proposition 3

$$
\prod_{j=1}^{k} \lambda_{j}^{\downarrow}(G) \leq \prod_{j=1}^{k} \lambda_{j}^{\downarrow}(L), \quad 1 \leq k \leq n .
$$


For $k=n$ there is equality here as $\operatorname{det}(G)=\operatorname{det}(L)$. This proves the first majorisation in (19).

To prove the second we start with the equation

$$
\sum_{j=1}^{m} w_{j}\left(A_{j} \# \Omega^{-1}\right)=I
$$

that the Wasserstein mean $\Omega$ satisfies. This follows from (3) and (10). Using the fact that the function $f(A)=A^{p}$ is operator concave for $0<p<1$ we obtain from (34) the inequality

$$
\sum_{j=1}^{m} w_{j}\left(A_{j} \# \Omega^{-1}\right)^{p} \leq I, \quad 0<p<1 .
$$

Using the arithmetic-geometric mean inequality (11) we get from this

$$
G\left(w ;\left(A_{1} \# \Omega^{-1}\right)^{p}, \ldots,\left(A_{m} \# \Omega^{-1}\right)^{p}\right) \leq I, \quad 0<p<1,
$$

and then using (29) we get

$$
G\left(w ;\left(\Lambda^{k} A_{1} \# \Lambda^{k} \Omega^{-1}\right)^{p}, \ldots,\left(\Lambda^{k} A_{m} \# \Lambda^{k} \Omega^{-1}\right)^{p}\right) \leq I, 0<p<1 .
$$

Now suppose $A_{1}, \ldots, A_{m}$ are such that $\Lambda^{k} \Omega \leq I$. Then $\Lambda^{k} \Omega^{-1} \geq I$, and hence $\Lambda^{k} A_{j} \# \Lambda^{k} \Omega^{-1} \geq \Lambda^{k} A_{j} \# I=\Lambda^{k} A_{j}^{1 / 2}$. Hence for all $0<p<1$ we have from the Löwner-Heinz inequality that

$$
\Lambda^{k}\left(A_{j} \# \Omega^{-1}\right)^{p}=\left(\Lambda^{k}\left(A_{j} \# \Omega^{-1}\right)\right)^{p}=\left(\Lambda^{k} A_{j} \# \Lambda^{k} \Omega^{-1}\right)^{p} \geq\left(\Lambda^{k} A_{j}^{1 / 2}\right)^{p}=\Lambda^{k} A_{j}^{p / 2} .
$$

Together with (35) this gives

$$
G\left(w ; \Lambda^{k} A_{1}^{p / 2}, \ldots, \Lambda^{k} A_{m}^{p / 2}\right) \leq \Lambda^{k} G\left(w ;\left(A_{1} \# \Omega^{-1}\right)^{p}, \ldots,\left(A_{m} \# \Omega^{-1}\right)^{p}\right) \leq I
$$

for all $0<p<1$. In other words,

$$
\Lambda^{k} G\left(w ; A_{1}^{p / 2}, \ldots, A_{m}^{p / 2}\right) \leq I, \quad 0<p<1 .
$$

Raise both sides to their $2 / p$ power to get

$$
\Lambda^{k} G\left(w ; A_{1}^{p / 2}, \ldots, A_{m}^{p / 2}\right)^{2 / p} \leq I, \quad 0<p<1 .
$$

Now let $p \rightarrow 0$ and use Proposition 3. This shows that $\Lambda^{k} L \leq I$.

We have shown that for $1 \leq k \leq n$, the condition $\Lambda^{k} \Omega \leq I$ implies that $\Lambda^{k} L \leq I$. From this we conclude that

$$
\lambda_{1}^{\downarrow}\left(\Lambda^{k} L\right) \leq \lambda_{1}^{\downarrow}\left(\Lambda^{k} \Omega\right),
$$

that is,

$$
\prod_{j=1}^{k} \lambda_{j}^{\downarrow}(L) \leq \prod_{j=1}^{k} \lambda_{j}^{\downarrow}(\Omega) .
$$

This proves the second part of Theorem 1.

The easiest part of the proof of Theorem 2 is that of the case $p=\infty$. Suppose $\Omega \leq I$. Then $I \leq \Omega^{-1}$, and by the monotonicity property of the geometric mean

$$
\sum_{j=1}^{m} w_{j}\left(A_{j} \# I\right) \leq \sum_{j=1}^{m} w_{j}\left(A_{j} \# \Omega^{-1}\right) .
$$


The left hand side equals $\sum_{j=1}^{m} w_{j} A_{j}^{1 / 2}$, and by (34) the right hand side equals $I$. So the inequality says $Q_{1 / 2}^{1 / 2} \leq I$, and hence $Q_{1 / 2} \leq I$. We have shown that $\Omega \leq I$ implies that $Q_{1 / 2} \leq I$. Hence

$$
\left\|Q_{1 / 2}\right\|_{\infty} \leq\|\Omega\|_{\infty}
$$

The proof we offer for $p=1$ is more intricate. Given $A_{1}, \ldots, A_{m}$ define for each $A \in \mathbb{P}$

$$
K(A)=A^{-1 / 2}\left(\sum_{j=1}^{m} w_{j}\left(A^{1 / 2} A_{j} A^{1 / 2}\right)^{1 / 2}\right)^{2} A^{-1 / 2} .
$$

It has been shown in [1] (see also Theorem 11 in [1]) that for every $S_{0} \in \mathbb{P}$ the sequence $S_{k+1}=K\left(S_{k}\right)$ converge to $\Omega$ and $\operatorname{tr} S_{k} \leq \operatorname{tr} S_{k+1} \leq \operatorname{tr} \Omega$ for all $k \geq 1$. The special choice $S_{0}=I$ gives $S_{1}=K(I)=Q_{1 / 2}$. Hence $\operatorname{tr} Q_{1 / 2} \leq \operatorname{tr} \Omega$, and therefore the inequality (24) is valid for $p=1$.

In the special case $m=2$ we have a simple proof. In this case $w=(1-t, t)$ for some $0<t<1$,

$$
\begin{aligned}
Q_{1 / 2}(w ; A, B) & =\left((1-t) A^{1 / 2}+t B^{1 / 2}\right)^{2} \\
& =(1-t)^{2} A+t^{2} B+(1-t) t\left(A^{1 / 2} B^{1 / 2}+B^{1 / 2} A^{1 / 2}\right),
\end{aligned}
$$

and $\Omega(w ; A, B)=A \diamond_{t} B$ as given in (4). So the inequality $\left\|Q_{1 / 2}\right\|_{1} \leq\|\Omega\|_{1}$ will be established if we can show that

$$
\operatorname{tr} A^{1 / 2} B^{1 / 2} \leq \operatorname{tr}(A B)^{1 / 2}
$$

Since $\operatorname{tr}(A B)^{1 / 2}=\operatorname{tr} A^{-1 / 2}(A B)^{1 / 2} A^{1 / 2}=\operatorname{tr}\left(A^{1 / 2} B A^{1 / 2}\right)^{1 / 2}$, this inequality can also be stated as

$$
\operatorname{tr} A^{1 / 4} B^{1 / 2} A^{1 / 4} \leq \operatorname{tr}\left(A^{1 / 2} B A^{1 / 2}\right)^{1 / 2} .
$$

This follows from Theorem IX.2.10 in [6].

To prove the last statement of Theorem 2 we have to show that

$$
\left\|Q_{1 / 2}(w ; A, B)\right\|_{2}^{2} \leq\|\Omega(w ; A, B)\|_{2}^{2}
$$

Using (37) and the cyclicity of trace, the left hand side of (39) is seen to be equal to

$$
\begin{aligned}
& \operatorname{tr}\left[(1-t)^{4} A^{2}+4(1-t)^{2} t^{2} A B+t^{4} B^{2}\right. \\
& \left.\quad+4(1-t)^{3} t A^{3 / 2} B^{1 / 2}+4(1-t) t^{3} A^{1 / 2} B^{3 / 2}+2(1-t)^{2} t^{2}\left(A^{1 / 2} B^{1 / 2}\right)^{2}\right] .
\end{aligned}
$$

To expand the right hand side we use the expression (44) for $\Omega$, cyclicity of trace, and the observation

$$
\begin{aligned}
\operatorname{tr} A(A B)^{1 / 2} & =\operatorname{tr} A A^{-1 / 2}(A B)^{1 / 2} A^{1 / 2} \\
& =\operatorname{tr} A\left(A^{1 / 2} B A^{1 / 2}\right)^{1 / 2} \\
& =\operatorname{tr}\left(A^{1 / 2} B A^{1 / 2}\right)^{1 / 2} A=\operatorname{tr}(B A)^{1 / 2} A .
\end{aligned}
$$


A little calculation shows that the right hand side of (39) is equal to

$$
\begin{aligned}
& \operatorname{tr}\left[(1-t)^{4} A^{2}+4(1-t)^{2} t^{2} A B+t^{4} B^{2}\right. \\
& +4(1-t)^{3} t A(A B)^{1 / 2}+4(1-t) t^{3} B(B A)^{1 / 2} \\
& \left.+2(1-t)^{2} t^{2}(A B)^{1 / 2}(B A)^{1 / 2}\right] .
\end{aligned}
$$

Thus to prove (31) we need two inequalities that are of independent interest and are stated in the following proposition.

Proposition 5. For all positive definite matrices $A$ and $B$ we have

$$
\begin{aligned}
& \text { (i) } \operatorname{tr}\left(A^{1 / 2} B^{1 / 2}\right)^{2} \leq \operatorname{tr}(A B)^{1 / 2}(B A)^{1 / 2}, \\
& \text { (ii) } \operatorname{tr} A^{3 / 2} B^{1 / 2} \leq \operatorname{tr} A(A B)^{1 / 2}
\end{aligned}
$$

Proof. We will show that

$$
\operatorname{tr}\left(A^{1 / 2} B^{1 / 2}\right)^{2} \leq \operatorname{tr} A B \leq \operatorname{tr}(A B)^{1 / 2}(B A)^{1 / 2} .
$$

The first inequality in (42) is the famous Lieb-Thirring inequality (see IX.62 in [6]). To prove the second note that

$$
\begin{aligned}
\operatorname{tr}(A B)^{1 / 2}(B A)^{1 / 2} & =\left\|(A B)^{1 / 2}\right\|_{2}^{2} \\
& \geq \sum_{j=1}^{n}\left[\lambda_{j}(A B)^{1 / 2}\right]^{2}=\sum_{j=1}^{n} \lambda_{j}(A B) \\
& =\operatorname{tr} A B .
\end{aligned}
$$

This proves the inequality (42).

The inequality (41) can be also be stated as

$$
\operatorname{tr} A^{3 / 4} B^{1 / 2} A^{3 / 4} \leq \operatorname{tr} A^{1 / 2}\left(A^{1 / 2} B A^{1 / 2}\right)^{1 / 2} A^{1 / 2} .
$$

We will prove a much stronger log majorisation:

$$
\lambda\left(A^{3 / 4} B^{1 / 2} A^{3 / 4}\right) \underset{\log }{\prec} \lambda\left(A^{1 / 2}\left(A^{1 / 2} B A^{1 / 2}\right)^{1 / 2} A^{1 / 2}\right) .
$$

We first prove

$$
\lambda_{1}^{\downarrow}\left(A^{3 / 4} B^{1 / 2} A^{3 / 4}\right) \leq \lambda_{1}^{\downarrow}\left(A^{1 / 2}\left(A^{1 / 2} B A^{1 / 2}\right)^{1 / 2} A^{1 / 2}\right) .
$$

As explained earlier, for this it suffices to prove the implication

$$
A^{1 / 2}\left(A^{1 / 2} B A^{1 / 2}\right)^{1 / 2} A^{1 / 2} \leq I \Longrightarrow A^{3 / 4} B^{1 / 2} A^{3 / 4} \leq I .
$$

This is equivalent to the statement

$$
\left(A^{1 / 2} B A^{1 / 2}\right)^{1 / 2} \leq A^{-1} \Longrightarrow B^{1 / 2} \leq A^{-3 / 2} .
$$

Here we invoke Furuta's inequality. This tells us that

$$
X \geq Y \geq 0 \Longrightarrow\left(X^{p+2 r}\right)^{1 / p} \geq\left(X^{r} Y^{p} X^{r}\right)^{1 / p}
$$

for all $p \geq 1, r \geq 0$. (See Corollary 4.4.2 in [7]). Choosing $X=A^{-1}, Y=$ $\left(A^{1 / 2} B A^{1 / 2}\right)^{1 / 2}, p=2, r=1 / 2$ in Furuta's inequality, we obtain the assertion (45). This gives us the inequality (44). 
Applying this to $k$ th antisymmetric tensor powers of the matrices involved we obtain

$$
\prod_{j=1}^{k} \lambda_{j}^{\downarrow}\left(A^{3 / 4} B^{1 / 2} A^{3 / 4}\right) \leq \prod_{j=1}^{k} \lambda_{j}^{\downarrow}\left(A^{1 / 2}\left(A^{1 / 2} B A^{1 / 2}\right)^{1 / 2} A^{1 / 2}\right),
$$

for $1 \leq k \leq n$. For $k=n$ there is equality here as both sides are equal to $\operatorname{det}\left(A^{3 / 2} B^{1 / 2}\right)$. This proves (43), and as a corollary (41).

All the assertions of Theorem 2 have been established.

\section{REMARKS}

The reader would have noticed that our Conjecture 1 asserts only weak majorisation and not weak log majorisation in (25). In [4] Audenaert has shown the following determinant inequality in the case $m=2$ and equal weights $\left(w_{1}, w_{2}\right)=(1 / 2,1 / 2)$,

$$
\operatorname{det} \Omega(A, B) \leq \operatorname{det} Q_{1 / 2}(A, B) .
$$

This goes in the direction opposite to (25), and opposite to what weak log majorisation would have implied.

It is reasonable to conjecture that the inequality (46) remains true in the several variable case as well.

Theorem 2 supplements some results proved in [12. In their analysis of the Cartan mean, Lim and Palfia [19] introduced another version of the power mean. They showed that for $0<t<1$, the equation

$$
X=\sum_{j=1}^{m} w_{j}\left(X \#_{t} A_{j}\right)
$$

has a unique positive definite solution. Call this $P_{t}\left(w ; A_{1}, \ldots, A_{m}\right)$. When $A_{1}, \ldots, A_{m}$ commute, $P_{t}=\left(\sum_{j=1}^{m} w_{j} A_{j}^{t}\right)^{1 / t}=Q_{t}$. Lim and Palfia showed that

$$
\lim _{p \rightarrow 0} P_{t}\left(w ; A_{1}, \ldots, A_{m}\right)=G\left(w ; A_{1}, \ldots, A_{m}\right) .
$$

Compare this with (21).

It was conjectured in [12] that

$$
\left\|\mid P_{t}\left(w ; A_{1}, \ldots, A_{m}\right)\right\| \leq\|\| Q_{t}\left(w ; A_{1}, \ldots, A_{m}\right)\|\|,
$$

for all $0<t<1$. The case $\|\cdot\|_{\infty}$ of this had been proved in [20] and the case $\|\cdot\|_{1}$ was proved in [12]. The case $0<p<\infty$ has been proved recently in [13]. Explicit formulas for $P_{t}$ are known only when $m=2$. In the special case of equal weights $\left(w_{1}, w_{2}\right)=(1 / 2,1 / 2)$ we have

$$
P_{1 / 2}(A, B)=\frac{1}{4}(A+B+2(A \# B)) .
$$

So in this special case the conjecture (49) says

$$
\|\| A+B+2(A \# B) \mid\|\leq\|\left\|A+B+A^{1 / 2} B^{1 / 2}+B^{1 / 2} A^{1 / 2}\right\| \| .
$$


In addition to the $\|\cdot\|_{p}$ norms, $p=1, \infty$, this was shown to be true for $p=2$ in [12]. Compare the statements (26) and (51).

In the course of the proofs in [12] certain log majorisations complementary to (43) have been proved. Together these say

$$
\lambda\left(A^{2}\left(A^{-1} B\right)^{1 / 2}\right) \underset{\log }{\prec} \lambda\left(A^{3 / 2} B^{1 / 2}\right) \underset{\log }{\prec} \lambda\left(A(A B)^{1 / 2}\right) .
$$

This implies the trace inequalities

$$
\operatorname{tr} A^{2}\left(A^{-1} B\right)^{1 / 2} \leq \operatorname{tr} A^{3 / 2} B^{1 / 2} \leq \operatorname{tr} A(A B)^{1 / 2} .
$$

These inequalities illustrate the effect of rearranging factors in noncommutative products. There are several inequalities of this kind that are known, the most famous being the Golden-Thompson and the Lieb-Thirring inequalities.

In view of the comparison between $L$ and $\Omega$ given in (19) and between $Q_{1 / 2}$ and $\Omega$ conjectured in (25), we may ask how $L$ and $Q_{1 / 2}$ compare with each other, It has been shown in [8] that for each $1 \leq j \leq n$, $\lambda_{j}^{\downarrow}\left(Q_{p}\left(w ; A_{1}, \ldots, A_{m}\right)\right)$ is a monotonically increasing function of $p$ on $(-\infty, \infty)$. In particular, using (21) we see from this

$$
\lambda_{j}^{\downarrow}\left(L\left(w ; A_{1}, \ldots, A_{m}\right)\right) \leq \lambda_{j}^{\downarrow}\left(Q_{1 / 2}\left(w ; A_{1}, \ldots, A_{m}\right)\right), \quad 1 \leq j \leq n .
$$

\section{ACKNOWLEDGEMENTs.}

The first author is a J. C. Bose National Fellow. The work of Y. Lim was supported by the National Research Foundation of Korea (NRF) grant funded by the Korea government(MEST) No.2015R1A3A2031159 and 2016R1A5A1008055.

\section{REFERENCES}

[1] P. C. Alvarez-Esteban, E. del Barrio, J. A. Cuesta-Albertos and C. Matran, A fixed point approach to barycenters in Wasserstein spaces, J. Math. Anal. Appl. 441 (2016), 744-762.

[2] T. Ando, On some operator inequalities, Math. Ann. 279 (1987), 157-159.

[3] T. Ando and F. Hiai, Log majorization and complementary Golden-Thompson type inequalities, Linear Algebra Appl. 197/198 (1994), 113-131.

[4] K. M. R. Audenaert, A determinantal inequality for the geometric mean with an application in diffusion tensor imaging, arXiv: 1502.06902.

[5] K. V. Bhagwat and R. Subramanian, Inequalities between means of positive operators, Math. Proc. Camb. Phil. Soc. 83 (1978), 393-401.

[6] R. Bhatia, Matrix Analysis, Springer, 1997.

[7] R. Bhatia, Positive Definite Matrices, Princeton University Press, 2007.

[8] R. Bhatia and P. Grover, Norm inequalities related to the matrix geometric mean, Linear Algebra Appl. 437 (2012), 726-733.

[9] R. Bhatia and J. Holbrook, Riemannian geometry and matrix geometric means, Linear Algebra Appl. 413 (2006), 594-618.

[10] R. Bhatia and R. L. Karandikar, Monotonicity of the matrix geometric mean, Math. Ann. 353 (2012), 1453-1467.

[11] R. Bhatia, T. Jain and Y. Lim, On the Bures-Wasserstein distance bewteen positive definite matrices, to appear in Expositiones Mathematicae.

[12] R. Bhatia, Y. Lim and T. Yamazaki, Some norm inequalities for matrix means, Linear Algebra Appl. 501 (2016), 112-122. 
[13] T. Dinh, R. Dumitru and J. Franc, On a conjecture of Bhatia, Lim and Yamazaki, Linear Algebra Appl. 532 (2017), 140-145.

[14] J. I. Fujii, M. Fujii and Y. Seo, The Golden-Thompson-Segal type inequalities related to the weighted geometric mean due to Lawson-Lim, J. Math. Inequal. 3 (2009), 511-518.

[15] F. Hansen, Means and concave products of positive semidefinite matrices, Math. Ann. 264 (1983), 119-128.

[16] F. Hansen and G. K. Pedersen, Jensen's inequality for operators and Löwner's theorem, Math. Ann. 258 (1982), 229-241.

[17] F. Hiai and D. Petz, Riemannian metrics on positive definite matrices related to means. II, Linear Algebra Appl. 436 (2012), 2117-2136.

[18] J. Lawson and Y. Lim, Monotonic properties of the least squares mean, Math. Ann. 351 (2011), 267-279.

[19] Y. Lim and M. Palfia, Matrix power means and the Karcher mean, J. Funct. Anal. 262 (2012), 1498-1514.

[20] Y. Lim and T. Yamazaki, On some inequalities for the matrix power and Karcher means, Linear Algebra Appl. 438 (2013), 1293-1304.

[21] M. Moakher, A differential geometric approach to the geometric mean of symmetric positive-definite matrices, SIAM J. Matrix Anal. Appl. 26 (2005), 735-747.

[22] A. Takatsu, Wasserstein geometry of Gaussian measures, Osaka J. Math. 48 (2011), 1005-1026.

[23] T. Yamazaki, The Riemannian mean and matrix inequalities related to the Ando-Hiai inequality and chaotic order, Oper. Matrices 6 (2012), 577-588.

Ashoka University, Sonepat, Haryana, 131029, India

E-mail address: rajendra.bhatia@ashoka.edu.in

Indian Statistical Institute, New Delhi 110016, India

E-mail address: tanvi@isid.ac.in

Department of Mathematics, Sungkyunkwan University, Suwon 440-746, KOREA

E-mail address: ylim@skku.edu 